\title{
SPG20, a novel biomarker for early detection of colorectal cancer, encodes a regulator of cytokinesis
}

\author{
GE Lind ${ }^{1,2,9}$, C Raiborg ${ }^{2,3,9}$, SA Danielsen ${ }^{1,2}$, TO Rognum ${ }^{4,5}$, E Thiis-Evensen ${ }^{6}$, G Hoff ${ }^{7}$, \\ A Nesbakken ${ }^{2,8}$, H Stenmark ${ }^{2,3}$ and RA Lothe ${ }^{1,2}$ \\ ${ }^{1}$ Department of Cancer Prevention, Institute for Cancer Research, Radiumhospitalet, Oslo University Hospital, Oslo, Norway; \\ ${ }^{2}$ Centre for Cancer Biomedicine, Faculty of Medicine, University of Oslo, Oslo, Norway; ${ }^{3}$ Department of Biochemistry, Institute \\ for Cancer Research, Radiumhospitalet, Oslo University Hospital, Oslo, Norway; ${ }^{4}$ Institute of Forensic Medicine, Rikshospitalet, \\ Oslo University Hospital, Oslo, Norway; ${ }^{5}$ Faculty of Medicine, University of Oslo, Oslo, Norway; ${ }^{6}$ Department of Medicine, \\ Rikshospitalet, Oslo University Hospital, Oslo, Norway; ${ }^{7}$ Department of Medicine Division of Gastroenterology, Telemark Hospital, \\ Skien, Norway and ${ }^{8}$ Surgical Department, Aker Hospital, Oslo University Hospital, Oslo, Norway
}

Colorectal cancer is a common disease with high mortality. Suitable biomarkers for detection of tumors at an early curable stage would significantly improve patient survival. Here, we show that the SPG20 (spastic paraplegia-20) promoter, encoding the multifunctional Spartin protein, is hypermethylated in $89 \%$ of colorectal carcinomas, $78 \%$ of adenomas and only $1 \%$ of normal mucosa samples. SPG 20 methylation was also present in a pilot series of stool samples and corresponding tumors from colorectal cancer patients. SPG20 promoter hypermethylation resulted in loss of mRNA expression in various cancer types and subsequent depletion of Spartin. We further showed that Spartin downregulation in cancer cells resulted in cytokinesis arrest, which was reversed when $S P G 20$ methylation was inhibited. The present study identifies $S P G 20$ promoter hypermethylation as a biomarker suitable for non-invasive detection of colorectal cancer, and a possible mechanism for cytokinesis arrest in colorectal tumorigenesis.

Oncogene (2011) 30, 3967-3978; doi:10.1038/onc.2011.109; published online 18 April 2011

Keywords: colorectal neoplasia; cytokinesis; early detection; methylation; Spartin; SPG20

\section{Introduction}

Colorectal cancer is the second and third most common cause of cancer deaths among men and women in Europe and the United States, respectively (Ferlay et al., 2007; Jemal et al., 2009). The survival of colorectal cancer patients is strongly associated with the tumor stage at the time of diagnosis. Patients with metastasis to distant organs have a poor 5-year survival (11\%), whereas most patients with a localized disease have

Correspondence: Proffesor RA Lothe, Department of Cancer Prevention, Institute for Cancer Research, Radiumhospitalet, Oslo University Hospital, Montebello, Oslo 0310, Norway.

E-mail: rlothe@rr-research.no

${ }^{9}$ Shared first authors.

Received 17 November 2010; revised 15 February 2011; accepted 24 February 2011; published online 18 April 2011 good prognosis (90\%) (Jemal et al., 2009). As development of a colorectal carcinoma from an adenoma may take several years, there is a large window of opportunity to detect tumors at a curable stage. Early detection of cancer and of precursor lesions followed by surgical removal would significantly reduce the number of cancer deaths. Although the quality of existing diagnostics and screening programs for colorectal cancer continues to improve, the sensitivity and specificity of non-invasive tests are still suboptimal.

Epigenetic alterations are defined as non-sequencebased modifications that are inherited through cell division (Feinberg et al., 2006). In the last decade such changes have been recognized to be at least as common as genetic changes in human cancer (Jones and Baylin, 2002). DNA methylation is the best studied epigenetic mechanism, and the presence of too much methylation (hypermethylation) in gene promoters may disrupt function by reducing or silencing gene expression. In colorectal cancer, several hypermethylated target genes have been identified in malignant tumors as well as in early benign precursor lesions (Lind et al., 2007; reviewed by Zitt et al. (2007) and Kim et al. (2010)). If such targets additionally showed high sensitivity and specificity for colorectal tumors, they would be suitable for early detection of colorectal cancer.

In a previous epigenome study, we identified a list of genes that potentially could be inactivated owing to promoter hypermethylation in colorectal cancer (Lind et al., 2006). One of these candidates, SPG20 (spastic paraplegia-20), has been analyzed in the present study for its suitability as a biomarker in colorectal cancer and for its functional mechanism.

The SPG20 gene is located in chromosome band $13 q 13.3$ and a one base deletion in exon-4 (1110delA) resulting in loss of expression of the mutated protein has been shown to cause the autosomal recessive spastic paraplegia-20 (Troyer syndrome) (Patel et al., 2002; Bakowska et al., 2008). The hereditary spastic paraplegias describe a group of inherited neurological disorders characterized by progressive muscle stiffness (spasticity) and development of paralysis (paraplegia) of the lower limbs (Proukakis et al., 2004). 
SPG20 encodes Spartin, which contains a Microtubule-Interacting and Trafficking molecule (MIT) domain in the $\mathrm{N}$-terminus and sequence similarities with a number of uncharacterized plant proteins in the C-terminus (Ciccarelli et al., 2003). Spartin is a multifunctional protein and has previously been found to be involved in intracellular epidermal growth factor receptor trafficking (Bakowska et al., 2007) and in lipid droplet turnover (Eastman et al., 2009; Hooper et al., 2010). It has also been reported to be an adaptor for the homologous to the E6-AP carboxyl terminus (HECT) domain-containing E3 ubiquitin ligases (Edwards et al., 2009; Milewska et al., 2009; Hooper et al., 2010) and an inhibitor of bone morphogenic protein (BMP) signaling (Tsang et al., 2009).

In the present study, the promoter methylation status of the SPG20 gene was determined in test and validation series of colorectal carcinomas, adenomas and normal mucosa. The methylation status was compared with mRNA expression levels in several cancer cell lines of various types. We show that the SPG20 promoter is hypermethylated in almost all colorectal carcinomas and adenomas, causing the expression of Spartin to be undetectable, whereas there is no hypermethylation of $S P G 20$ in normal mucosa. Interestingly, we identify cytokinesis arrest as a functional consequence of Spartin downregulation, a condition thought to be associated with carcinogenesis. We therefore propose that SPG20 hypermethylation could represent both a mechanism and a biomarker in colorectal carcinogenesis.

\section{Results}

\section{Identification of SPG20 as a biomarker for colorectal cancer}

By using microarray gene expression profiling in combination with epigenetic drug treatment (5-aza$2^{\prime}$ deoxycytidine (AZA)) of colon cancer cell lines we have previously identified $S P G 20$ as a DNA methylation candidate in colorectal cancer (Lind et al., 2006). In the present study, we found that the promoter of the SPG20 gene was hypermethylated in 20/20 colon cancer cell lines. A test set comprising 74 colorectal carcinomas, 60 adenomas and 51 normal mucosa samples was analyzed by quantitative methylation-specific PCR (qMSP). $S P G 20$ was found to be methylated in $91 \%, 75 \%$ and
$2 \%$ of the samples, respectively. Likewise, in a clinically independent validation set consisting of 105 colorectal carcinomas, 51 adenomas and 59 normal mucosa samples, $S P G 20$ was methylated in $88 \%, 82 \%$ and $0 \%$ of the samples, respectively (Table 1 ). The methylation status of the SPG20 promoter showed no association with the MSI status of the analyzed carcinomas. None $(0 \%)$ of the normal mucosa samples taken in distance from the carcinomas $(n=105)$ was methylated. For the combined test and validation series, Receiver Operating Characteristics (ROC) curves showed an area under the curve (AUC) of $0.947 \quad(P=2.87 \mathrm{E}-37$; asymptotic 95\% confidence interval: $0.919-0.975)$ for carcinomas versus normal mucosa and $0.913(P=2.66 \mathrm{E}-26$; asymptotic $95 \%$ confidence interval: $0.872-0.954)$ for adenomas versus normal mucosa (Figure 1). SPG20 methylation was also detected in 6/9 (67\%) stool samples from patients with methylated carcinomas. From one patient two stool samples were taken, collected proximally and distally to the carcinoma during open surgery. Both the carcinoma and the distal stool sample were methylation-positive, whereas the proximal stool sample was unmethylated. Direct bisulfite sequencing of the SPG20 promoter in colon cancer cell lines confirmed the DNA methylation status as assessed by MSP (Supplementary Figure S1). These results indicate that the SPG20 promoter is hypermethylated in the majority of colorectal carcinomas and adenomas, but very rarely in normal colonic epithelium, and that SPG20 methylation is a suitable biomarker for non-invasive analysis.

\section{Real-time quantitative analysis shows that SPG20} promoter hypermethylation causes loss of gene expression In order to examine the relationship of aberrant promoter methylation with gene expression, cancer cell lines, colorectal carcinomas and normal mucosa samples were subjected to quantitative real-time PCR analysis. The level of mRNA expression of SPG20 was strongly associated with promoter methylation status in cancer cell lines $(P=2.0 \mathrm{E}-6$; Figure $2 \mathrm{a})$ and tissue samples $(P=4.0 \mathrm{E}-6$, normals and carcinomas; $P=0.008$, only carcinomas). Measurements of mRNA levels in six colon cancer cell lines treated with epigenetic drugs (AZA and trichostatin-A (TSA)) also indicated an association between $S P G 20$ promoter hypermethylation and gene expression. The mRNA levels of $S P G 20$ were

Table 1 Frequency and distribution of promoter methylation levels (PMR values) in normal colorectal mucosas, adenomas and carcinomas as measured by qMSP

\begin{tabular}{|c|c|c|c|c|c|c|c|}
\hline \multirow[t]{2}{*}{$S P G 20$} & \multicolumn{2}{|c|}{ Normal mисоsas } & \multicolumn{2}{|c|}{ Adenomas } & \multicolumn{2}{|c|}{ Carcinomas } & \multirow[t]{2}{*}{ P-value } \\
\hline & $\mathrm{n}(\%)$ & Median $(I Q R)$ & $\mathrm{n}(\%)$ & Median $(I Q R)$ & $\mathrm{n}(\%)$ & Median (IQR) & \\
\hline Test set & $1 / 51(2 \%)$ & $0.49(1.18)$ & $45 / 60(75 \%)$ & $20.17(33.13)$ & $67 / 74(91 \%)$ & $44.77(47.15)$ & $5.32 \mathrm{E}-$ \\
\hline Validation set & $0 / 59(0 \%)$ & $1.88(2.13)$ & $42 / 51(82 \%)$ & $29.25(47.08)$ & $92 / 105(88 \%)$ & $37.29(35.41)$ & $9.27 \mathrm{E}-24$ \\
\hline Combined (test and validation) & $1 / 110(1 \%)$ & $1.17(2.36)$ & $87 / 111(78 \%)$ & $23.97(33.85)$ & $159 / 179(89 \%)$ & $39.21(39.84)$ & $1.33 \mathrm{E}-4$ \\
\hline
\end{tabular}

Abbreviations: IQR, interquartile range (75th percentile-25th percentile); $n$, number of positive cases; PMR, percentage methylated reference; qMSP, quantitative methylation-specific PCR; SPG20, spastic paraplegia-20.

${ }^{\text {a}}$ Kruskal-Wallis test. 


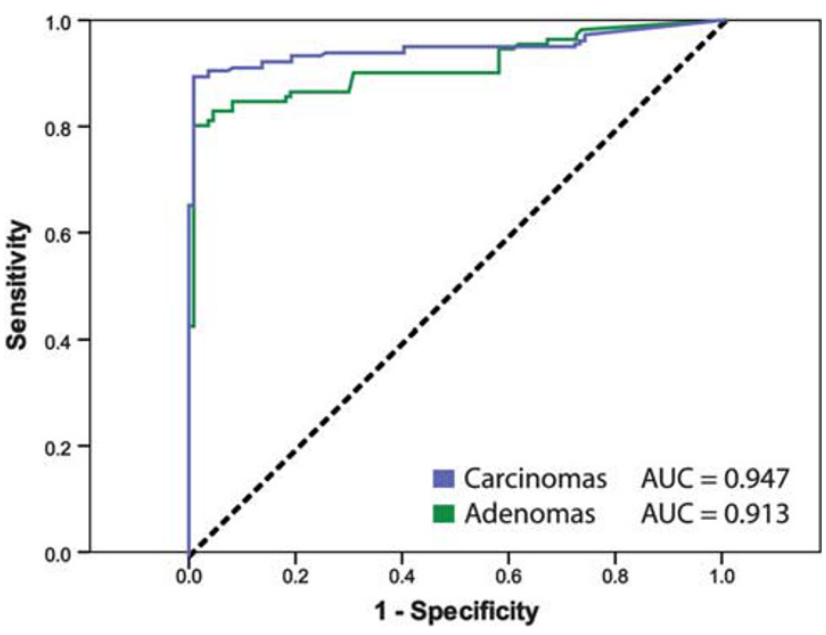

Figure 1 ROC curves show that $S P G 20$ is a biomarker for colorectal carcinomas and adenomas. The area under the ROC curve (AUC) signifies the accuracy of the SPG20 biomarker for distinguishing colorectal carcinomas (blue line) and adenomas (green line) from normal colorectal tissue samples. AUC, area under the curve; ROC, receiver operating characteristics; SPG20, spastic paraplegia-20.

upregulated in five of the six methylated cell lines after drug treatment (Figure 2b). These analyses show that the promoter hypermethylation found in colon cancer reduces the cellular levels of $S P G 20$ mRNA.

\section{Hypermethylated colon cancer cell lines do not express the SPG20 protein product Spartin}

As the hypermethylated cell lines did not express $S P G 20$ mRNA, we wanted to confirm this transcription deficiency on the protein level. The SPG20 gene encodes the 666-amino-acid (80-kDa) protein, Spartin (Bakowska et al., 2005; Robay et al., 2006). In addition to the normal colon epithelial cell line CRL-1790, four colon cancer lines with hypermethylated SPG20 promoter were analyzed: two microsatellite unstable (MSI; HCT116 and RKO) and two microsatellite stable (MSS; SW480 and HT29) lines. As expected, they did not express detectable amounts of Spartin by western blotting or by immunofluorescence confocal microscopy, as opposed to the control line (Figure 3), confirming the results from the mRNA analysis.

Spartin localizes to the spindle poles and the cytokinesis bridge of dividing daughter cells

Spartin is a multifunctional protein with a broad subcellular localization pattern (Lu et al., 2006; Robay et al., 2006; Bakowska et al., 2007; Eastman et al., 2009; Edwards et al., 2009; Tsang et al., 2009; Renvoise et al., 2010). In addition to diffuse cytosolic staining, we could clearly see that Spartin localized to the cytokinesis bridge of dividing daughter cells in CRL-1790 cells (Figure 3c, arrow in panel b). As the CRL-1790 cells had very few cytokinesis profiles, we continued our studies on the localization of endogenous Spartin in HeLa and hTERT RPE-1 cells. We could observe a very similar localization pattern in these cells, with diffuse cytosolic staining colocalizing with $\alpha$-tubulin in interphase cells (Supplementary Figure S2) and localization to the cytokinesis bridge of late cytokinesis profiles. In prometaphase cells, Spartin showed very strong distribution at the spindle poles in HeLa, CRL-1790 (Figures $4 \mathrm{~g}-\mathrm{k}$ ) and hTERT RPE-1 (Supplementary Figure S3) cells but not in the four colon cancer cell lines (Supplementary Figure S4).

Colon cancer cell lines contain several cytokinesis profiles The immunofluorescence confocal microscopy analyses suggested that the various cell lines had different growth rates (Figures 3b-g). We consequently analyzed the relative proliferation rates and found that the colon cancer cell lines proliferated four to 13 times faster than the control line (Figure 5a).

The cells were simultaneously analyzed for the numbers of cytokinesis profiles by using the cytokinesis markers Aurora-B and $\alpha$-tubulin (Carmena and Earnshaw, 2003; Steigemann and Gerlich, 2009). All four cell lines had a significantly higher number of cells in cytokinesis than the control line (Figure 5b). Interestingly, we could observe a correlation between proliferation rate and the number of cytokinesis bridges. The cell lines with the highest proliferation rate (HCT116 and RKO) showed a moderate increase in the number of cells in cytokinesis, whereas the slower growing cell lines (HT29 and SW480) showed a high number of cells interconnected by a cytokinesis bridge (Figure 5). Taken together, these observations indicate a delay in cytokinesis in HT29 and SW480.

Colon cancer cell lines have convoluted cytokinesis bridges Many of the cytokinesis bridges in HT29 and SW480 were kinked, nicked or convoluted, indicating problems with the final abscission step (Figure 6). This was particularly noticeable in SW480. Bridges that appeared to be normal often turned out to be kinked when watched in a side view in three-dimensional reconstructions from confocal z-stack images (Supplementary Figure S5). In many of the cytokinesis profiles, the localization of Aurora-B was dispersed along the length of the bridge (Figures 6a, c and e, and Supplementary Figure S5), indicating that these cells were in a very late stage of cytokinesis, when Aurora-B is transported back toward the cell body (Murata-Hori et al., 2002). Occasionally we observed three cells linked together through cytokinesis bridges, resembling pearls on a string (Supplementary Figure S6). Interestingly, we also observed examples of DNA-containing intercellular bridges in these cells (Supplementary Figure S7). In contrast to the slower growing HT29 and SW480, the highly proliferative HCT116 and RKO cells and the CRL-1790 control cells showed more normal-looking cytokinesis bridges (Figures $5 \mathrm{c}$ and $\mathrm{d}$, and Supplementary Figure S5). Taken together, these observations indicate that SPG20-hypermethylated colon cancer cell lines are hampered in their completion of cytokinesis. 
siRNA-mediated depletion of Spartin results in late cytokinesis arrest and convoluted cytokinesis bridges in HeLa and hTERT RPE-1 cells

To directly address a possible role of Spartin in cytokinesis, we performed RNA interference experiments using HeLa and hTERT RPE-1 cells. Western blotting and immunofluorescence analysis indicated an efficient and stable knockdown of Spartin (Supplementary Figures S8, S9A, and S10A). We next performed quantitation of different mitotic stages in control and Spartin-knockdown cells. Whereas there was no difference between control and short interfering RNA (siRNA)-treated cells in the early stages of mitosis, there was a significant increase in the number of cells in late cytokinesis upon Spartin depletion (oligo1: $P=0.0029 ; \quad$ oligo 2: $P=0.001 ;$ independent-samples $t$-test; Supplementary Figures S9B and S10B). Moreover, Spartin-depleted HeLa and hTERT RPE-1 cells showed a significantly slower growth rate compared with control cells (Supplementary Figure S9C), indicating a correlation between growth rate and cytokinesis arrest similar to HT29 and SW480. Intriguingly, we could observe convoluted cytokinesis bridges in the Spartin-depleted cells (Supplementary Figures S9D and E, and S10C-E), identical to the morphology of HT29 and SW480. Aurora-B localized along the

a

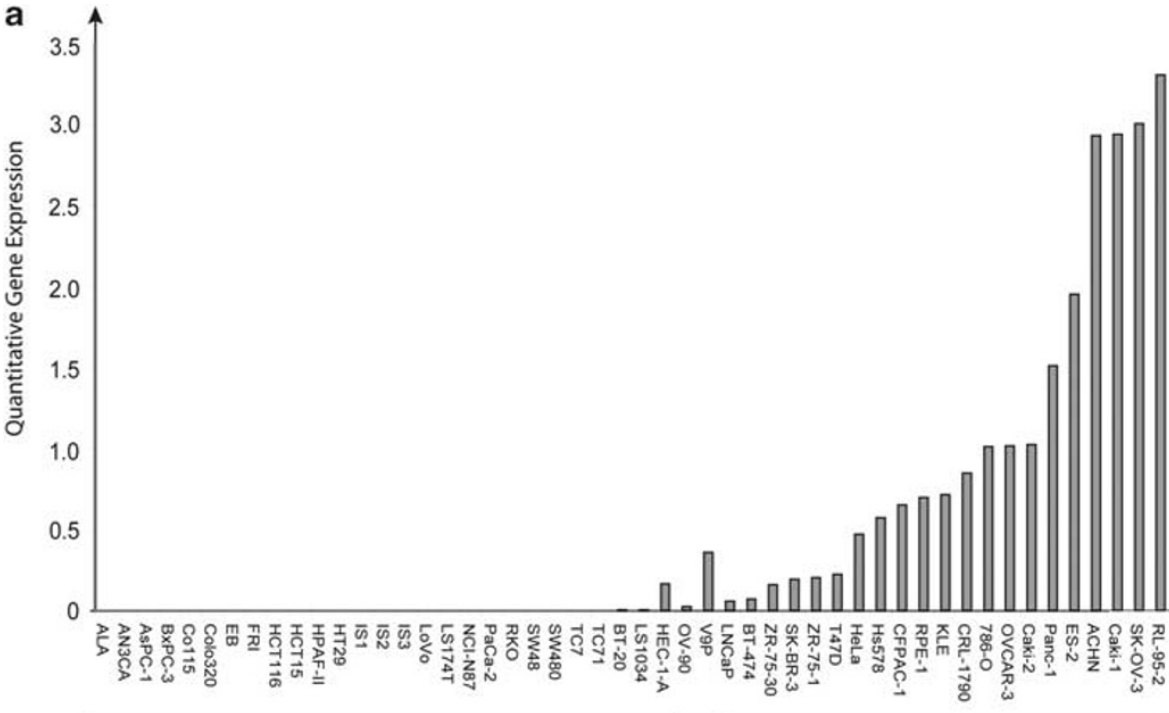

Hypermethylated

Partially methylated

Unmethylated

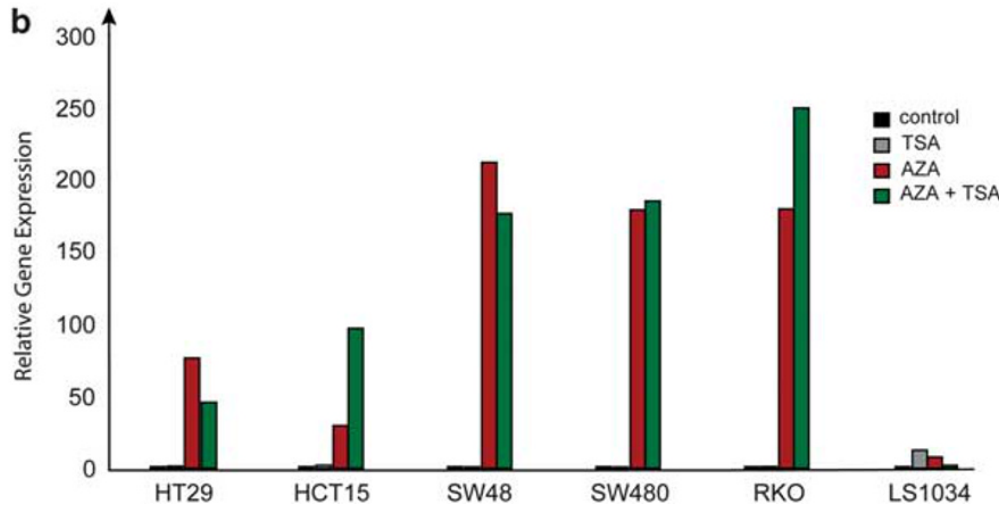

Figure 2 Promoter methylation of $S P G 20$ is associated with loss of gene expression. (a) Gene expression and DNA promoter methylation status of $S P G 20$ in cell lines. The quantitative gene expression levels are shown as ratios between the median of $S P G 20$ and the average of two endogenous controls, GUSB and ACTB. (b) Relative gene expression of SPG20 in colon cancer cell lines treated with $1 \mu \mathrm{M}$ AZA for $72 \mathrm{~h}, 0.5 \mu \mathrm{M}$ TSA for $12 \mathrm{~h}$ and a combination of both drugs (AZA + TSA). AZA, 5-aza-2'deoxycytidine; $S P G 20$, spastic paraplegia-20; TSA, trichostatin-A.

Figure 3 The SPG20-encoded protein Spartin is not expressed in hypermethylated colon cancer cell lines. (a) Western blot analysis showing levels of Spartin expression in colon epithelial cell lines. (b-g) Immunofluorescence confocal images of endogenous Spartin (red) in the indicated cell lines. The pictures in panels $\mathbf{b}$ and $\mathbf{d}-\mathbf{g}$ are generated using identical microscopy settings for comparison of the Spartin level. The arrow points to a cytokinesis bridge (enlarged in panel c). The inset in panel c shows an overlay with phase-contrast image to visualize the midbody. Nuclei are shown in blue. The cells are fixed with 3\% PFA. Size bars: $20 \mu \mathrm{m}$. PFA, paraformaldehyde; $S P G 20$, spastic paraplegia-20. 
Novel cancer biomarker and function: SPG20-Spartin GE Lind et al
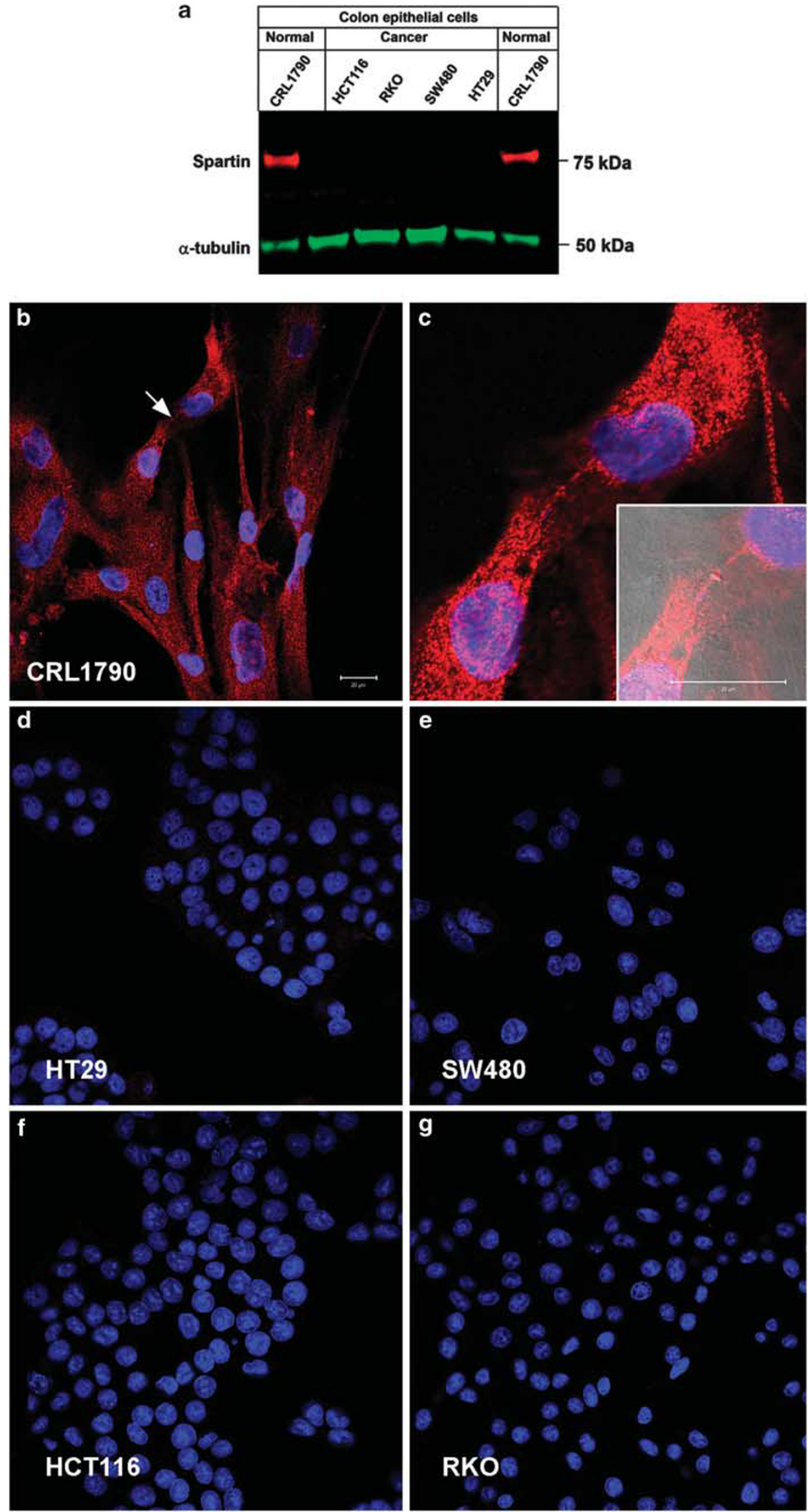

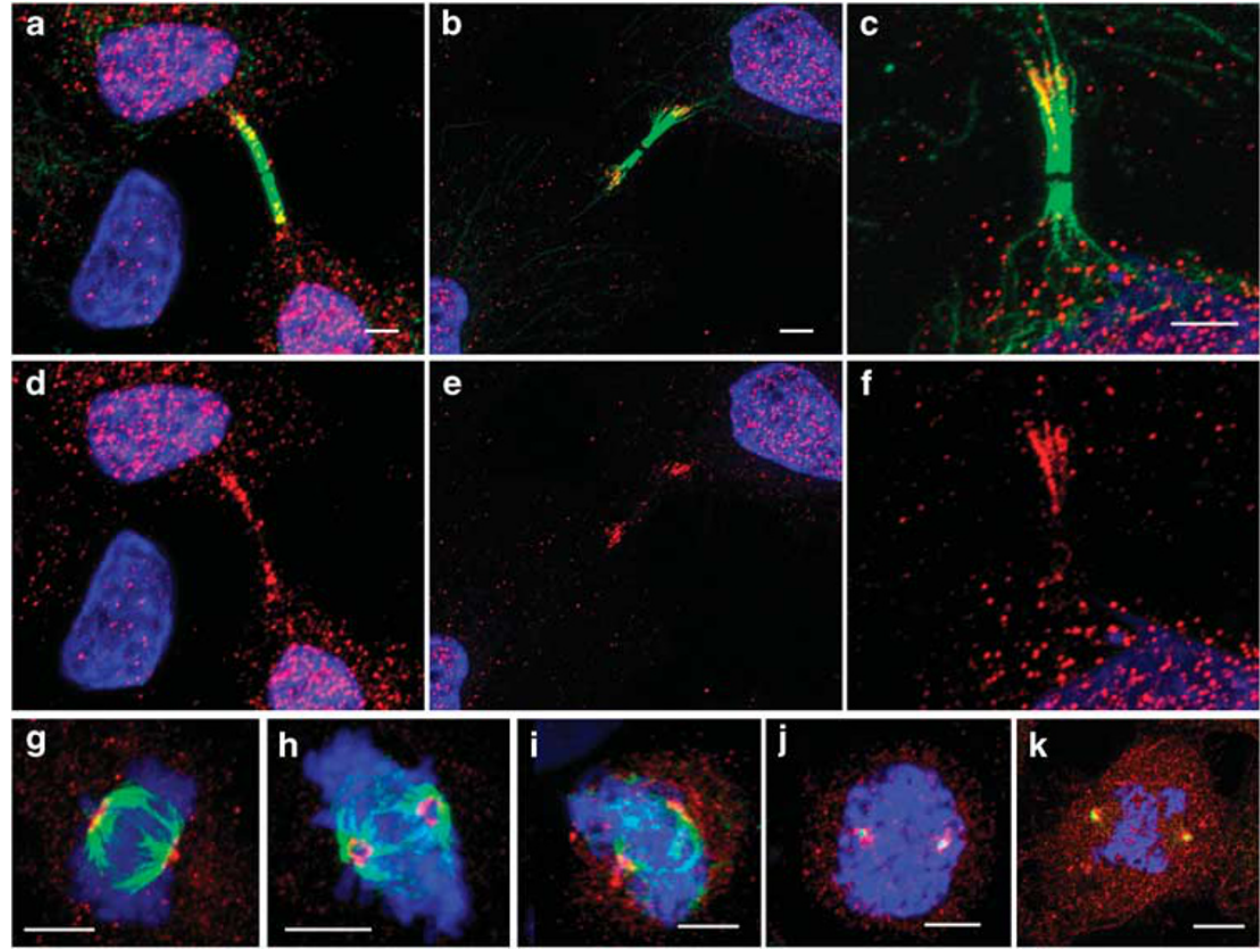

Figure 4 Spartin localizes to the spindle poles and the cytokinesis bridge of dividing cells. Three-dimensional reconstructions of immunofluorescence confocal images showing the localization of endogenous Spartin (red) to the cytokinesis bridge of HeLa cells (a-f) and to the spindle poles of prometaphase in HeLa $(\mathbf{g}, \mathbf{h}, \mathbf{k})$ and CRL-1790 (i, j) cells. $\alpha$-Tubulin $(\mathbf{a}-\mathbf{c}, \mathbf{g}-\mathbf{i})$ or $\gamma$-tubulin $(\mathbf{j}, \mathbf{k})$ is shown in green. Nuclei are shown in blue. Yellow indicates colocalization. The cells were pre-permeabilized with $0.05 \%$ saponin prior to fixation with $3 \%$ PFA $(\mathbf{a}-\mathbf{i})$ or fixed directly in methanol $(\mathbf{j}, \mathbf{k})$. Size bars: $5 \mu \mathrm{m}$. PFA, paraformaldehyde.
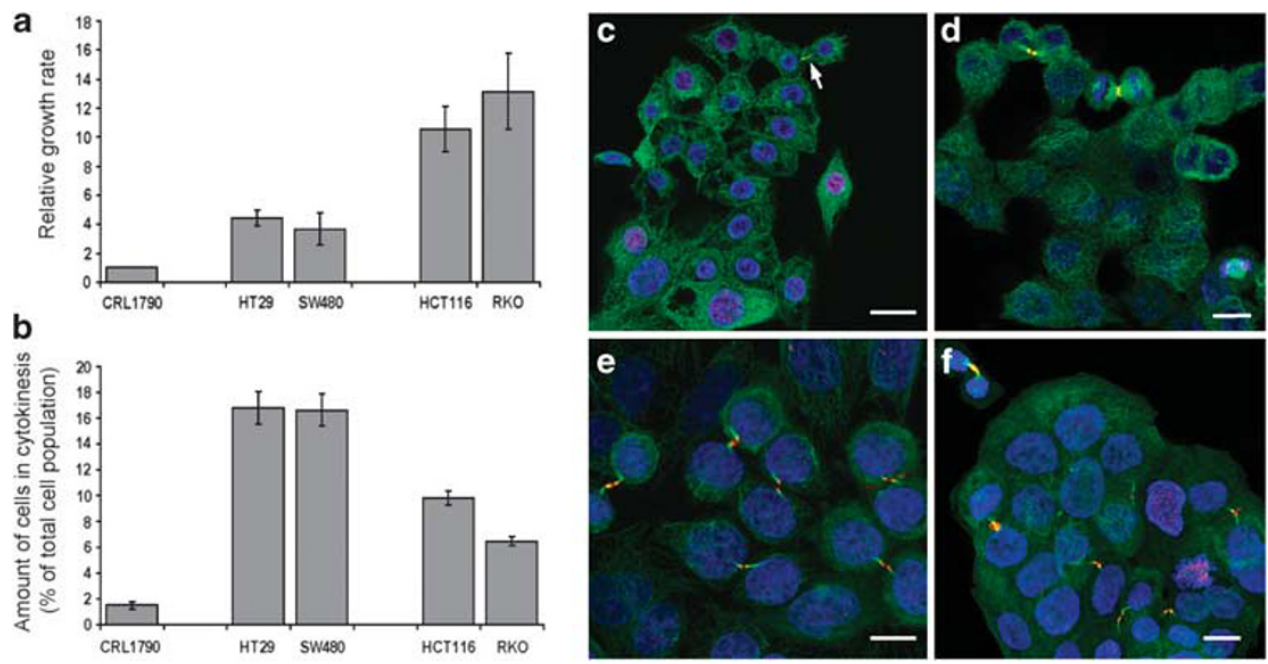

Figure 5 Colon cancer cell lines show several cytokinesis profiles. (a) The relative numbers of cells were quantified from immunofluorescence pictures using the Olympus Scan R microscope. (b) The cells were stained for $\alpha$-tubulin and Aurora-B to visualize cytokinesis profiles. The immunofluorescence pictures were used to quantify the percentage of cells in cytokinesis. The error bars show the \pm s.e.m. of four independent experiments. In total, more than 3000 cells were counted for the CRL-1790 control line, approximately 10000 for HT29, and SW480 and between 30000 and 40000 for the HCT116 and RKO lines. (c-f) Three-dimensional reconstructions of immunofluorescence confocal z-stack images showing the difference in the amount of cytokinesis profiles between the four colon cancer cell lines (c, RKO; d, HCT116; e, SW480; f, HT29). Aurora-B (red), $\alpha$-tubulin (green) and nuclei (blue). The arrow points to a cytokinesis profile. The cells were fixed with 3\% PFA. PFA, paraformaldehyde.

whole bridge, suggesting an arrest in very late cytokinesis. These results indicate that Spartin is required to complete cytokinesis in HeLa and hTERT RPE-1 cells, and the cytokinesis phenotype we observe in HT29 and SW480 is probably due to lack of Spartin in these cells.

An arrest in cytokinesis is often accompanied by an accumulation of binuclear cells (Fujiwara et al., 2005; 

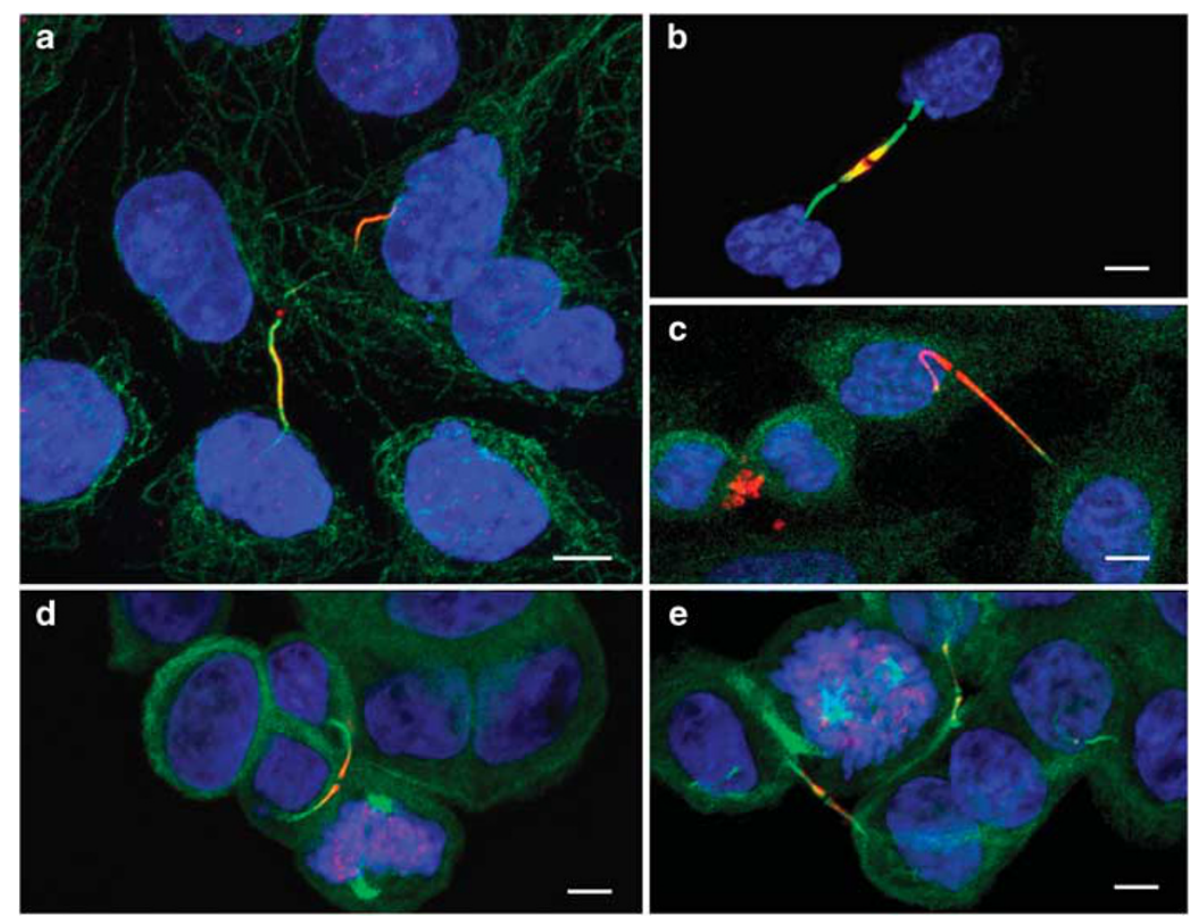

Figure 6 'Slower' growing colon cancer cell lines have convoluted cytokinesis bridges. A three-dimensional reconstruction of immunofluorescence confocal z-stack images showing the morphology of cytokinesis profiles in SW480 cells (a-c) and HT29 cells (d-e). Aurora B (red), $\alpha$-tubulin (green) and nuclei (blue). In addition to having convoluted cytokinesis bridges, both cell lines show distribution of Aurora-B along the bridge, indicative of late cytokinesis profiles. The cells were pre-permeabilized with $0.05 \%$ saponin prior to PFA fixation (a, b) or fixed directly with $3 \%$ PFA. Size bars, $5 \mu \mathrm{m}$. PFA, paraformaldehyde.

Ganem et al., 2007; Steigemann et al., 2009). Surprisingly, however, we were not able to detect any increase in the number of binuclear cells in the Spartin-depleted HeLa (Supplementary Figure S10B) and hTERT RPE-1 cells (data not shown). This might suggest that the cells become arrested at a very late stage of cytokinesis, at a point when regression of the cytokinesis furrow is no longer possible.

Rescue of Spartin expression results in a normalization of the cytokinesis process in HT29 colon cancer cells

To examine whether upregulation of Spartin expression in cancer cells could reverse the cytokinesis phenotype associated with SPG20 hypermethylation, we treated initially non-expressing HT29 colon cancer cells as well as CRL-1790 control cells with AZA. Both western blotting and immunofluorescence analysis confirmed the re-expression of moderate levels of Spartin in the HT29 cells after the treatment (Figure 7). AZA treatment had no influence on the number of cytokinesis profiles in CRL-1790 cells $(P=0.344)$ or hTERT RPE-1 cells (not shown). However, the number of HT29 cells in cytokinesis was significantly reduced after AZA treatment $(P=0.0008)$, indicating that re-expression of Spartin results in a normalization of cytokinesis and thereby prevents cytokinesis arrest. This was also underscored by the normal morphology of the midbody structures in the AZA-treated HT29 colon cancer cells. By contrast, non-Spartin-expressing HT29 cells showed several kinked, nicked or convoluted midbodies (Figure 6), indicating problems with the final abscission step. The AZA treatment did not affect the proliferation rate of either the HT29 colon cancer cells or the CRL1790 cells. These results are consistent with the idea that the cytokinesis arrest observed in HT29 colon cancer cells is due to $S P G 20$ hypermethylation and consequent downregulation of Spartin.

\section{Discussion}

In the present study we have used a quantitative highthroughput methylation analysis to show that the promoter of the SPG20 gene is hypermethylated in the vast majority of colorectal carcinomas and adenomas. The methylation was highly tumor-specific, underscored by lack of SPG20 methylation in normal colorectal mucosa samples. The findings have been validated in clinically independent sample series, altogether counting 505 tissue samples. Using ROC SPG20 methylation reached a very high area under the curve (AUC) value for carcinomas and for adenomas versus normal mucosa.

SPG20 methylation was also detected in a pilot series of stool samples from patients with colorectal carcinoma, underscoring its potential as a biomarker for early detection of colorectal cancer. Our finding that SPG20 promoter hypermethylation shuts down the expression of Spartin, resulting in cytokinesis arrest, suggests a 

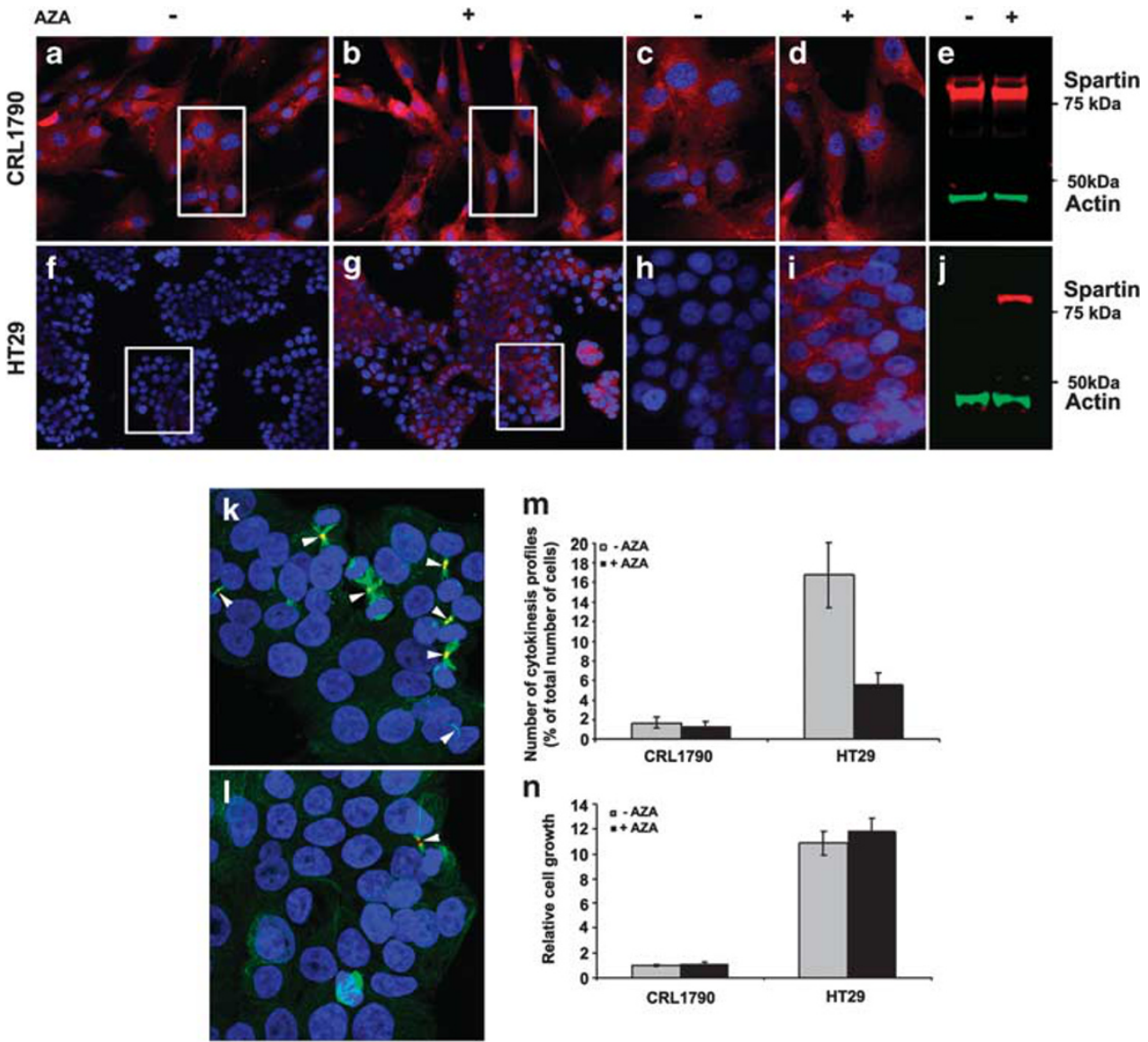

Figure 7 Rescue of Spartin expression results in the normalization of the cytokinesis process. Identical number of CRL-1790 cells (a-d) and HT29 cells (f-i) were seeded out on coverslips and treated or not with $1 \mu \mathrm{M}$ AZA for 3 days as indicated in the figure. The cells were fixed with 3\% PFA and stained for DNA (blue) and endogenous Spartin (red). (c, $\mathbf{d}, \mathbf{h}, \mathbf{i})$ Magnified views of the areas indicated by white squares. (e, j) Western blots showing endogenous Spartin in AZA-treated versus that in non-treated cells. HT29 cells were treated (l) or not (k) with AZA, fixed with 3\% PFA and stained for Aurora-B (red), $\alpha$-tubulin (green) and DNA (blue). The difference in the number of cytokinesis profiles between AZA-treated and non-treated cells (arrowheads $\mathbf{k}, \mathbf{l}$ ) is shown in panel $\mathbf{m}$. (n) Quantitations of relative growth rates. The error bars show the \pm s.d. of four independent coverslips. For each condition, approximately 3000 cells were quantified for CRL-1790 and 30000 cells for HT29. AZA, 5-aza-2'deoxycytidine; PFA, paraformaldehyde.

potential role for $S P G 20$ hypermethylation in genomic instability.

In contrast to genetic tests, which in most cases will require several parallel analyses in order to establish the mutation status of a single target gene (such as TP53), DNA promoter methylation occurs at a defined region of the gene and is easily analyzed in a single costeffective reaction, thereby demanding less biological starting material. Indeed, results from several studies of stool and serum DNA underscore the potential of using a non-invasive DNA methylation tests for early detection and/or screening for colorectal cancer (reviewed by Zitt et al. (2007) and Kim et al. (2010)). However, in spite of a few promising results (Müller et al., 2004; Chen et al., 2005; Zhang et al., 2007; Devos et al., 2009), specific and sensitive biomarkers to be included in an effective non-invasive test are still lacking. $S P G 20$ promoter hypermethylation has previously been shown in ovarian cancers and proposed as a methylated target in prostate cancer based on genome-wide analyses
(Goh et al., 2007; Kron et al., 2009). To the best of our knowledge, this is the first report identifying and validating the methylation status of SPG20 in a large cancer patient series, here shown for colorectal cancer. The resulting high sensitivity and specificity identifies $S P G 20$ as one of the most promising single biomarkers for early detection of colorectal cancer.

By using real-time quantitative gene expression analyses we showed that promoter hypermethylation of $S P G 20$ was associated with loss of gene expression. This was confirmed when the gene expression of $S P G 20$ was restored in originally hypermethylated and nonexpressing colon cancer cell lines by removal of DNA methylation using AZA. As expected, no Spartin protein expression could be seen in the hypermethylated colon cancer cell lines either by western blot analyses or by immunofluorescence confocal microscopy. Spartin expression was, however, seen in dividing control cells and in the unmethylated HeLa and hTERT RPE-1 cell lines where it, in accordance with previous findings, was 
located to the centrosome and midbody structures (Robay et al., 2006).

Cytokinesis is the final part of the M-phase of the cell cycle, where the cytoplasm is divided between two daughter cells. It is initiated by the assembly of a contractile ring, which constricts the cell membrane into generating a cleavage furrow. This ingresses until a structure of densely packed microtubules, the midbody, is formed. Subsequent cleavage of the midbody completes the cytokinesis (reviewed by Sagona and Stenmark (2010)). Microtubule severing during the abscission stage of cytokinesis is mediated by the AAA ATPase Spastin, which is found at the midbody during cytokinesis (Errico et al., 2004; Connell et al., 2009). Interestingly, Spastin contains an MIT domain that is closely related to that of Spartin (Ciccarelli et al., 2003). Spastin is encoded by the SPG4 gene, which is mutated in the most frequent form of autosomal dominant spastic paraplegia (Hazan et al., 1999). Thus, Spastin not only has structural similarity to Spartin, but also shares its association with spastic paraplegia. This opens the possibility that these two proteins could function in the same pathway.

Based on the midbody localization of Spartin as well as its common features with Spastin, we hypothesized that Spartin as well could have a role in cytokinesis. To provide insight into the underlying biology of SPG20 hypermethylation in cancer cells, we investigated cell proliferation in Spartin-deficient colon cancer cell lines and discovered that the 'slower' proliferating cell lines showed significantly more cytokinesis profiles and convoluted midbody structures compared with the highly proliferative cell lines. HeLa and hTERT RPE1 cells in which Spartin expression was knocked down by siRNA confirmed the phenotype seen among the 'slower' growing colon cancer cell lines by showing cytokinesis arrest as well as convoluted midbodies. Interestingly, a strikingly similar midbody morphology has been reported in cells with knockdown of Spastin (Connell et al., 2009). When the Spartin protein was reexpressed in initially non-expressing HT29 colon cancer cells, the number of cells arrested in late cytokinesis was significantly reduced. Taken together, these observations show that the multifunctional Spartin protein also has a role in cytokinesis. This novel role of the Spartin protein was recently also reported by Renvoise et al. (2010), who showed that Spartin colocalized with Ist1 (an endosomal sorting complex required for transport; ESCRT protein) at the midbody of HeLa cells and that depletion of Spartin with siRNA markedly impaired cytokinesis.

Failure of cytokinesis and subsequent aneuploidy has been shown to be associated with carcinogenesis (reviewed by Sagona and Stenmark, 2010). In cells experiencing cytokinesis arrest, a regression of the cytokinesis furrow can result in binuclearity (Fujiwara et al., 2005; Ganem et al., 2007; Steigemann et al., 2009). Although a significant increase in the number of multinucleated Spartin-depleted HeLa cells was reported by Renvoise et al. (2010), binuclear Spartindeficient cells were not observed in the present study either in HeLa cells or colon cancer cells. It is also worth noting that Spastin-deficient cells, similarly to Spartindeficient cells, show cytokinesis arrest without a binuclear phenotype (Connell et al., 2009), which further emphasizes the possible functional relationship between these two proteins. The lack of binuclear cells might suggest that the cells become arrested at a very late stage of cytokinesis where abortion of cytokinesis is no longer possible. The functional consequences of the cytokinesis arrest induced by Spartin downregulation therefore remain somewhat enigmatic. Cytokinesis failure in the absence of midbody regression has three alternative outcomes - an eventual (albeit delayed) midbody abscission, activation of apoptotic cell death ( $\mathrm{Bu}$ et al., 2008; Connell et al., 2009) or proliferation of cells that remain interconnected by thin intercellular bridges. Delayed abscission is associated with chromosomal instability (Fujiwara et al., 2005; Ganem et al., 2007), whereas interconnected cells tend to show synchronized responses to proliferation and differentiation cues (Ventelä et al., 2003; Vidulescu et al., 2004; Pointis et al., 2010). Both these mechanisms might thus contribute to carcinogenesis. Interestingly, both the 'slower' proliferating colon cancer cell lines included in the present study (HT29 and SW480) have a chromosomal unstable phenotype (Lengauer et al., 1997).

In the present study, we observed that, even though colon cancer cell lines with convoluted midbodies have a lower proliferation rate than cancer lines without cells arrested in late cytokinesis, they still proliferate significantly faster than control cells. This might be explained by the presence of multiple additional aberrations accumulated during tumorigenesis, which provides the cancer cells with a selective advantage. The nature of such additional aberrations might also explain why we observed a delay in cytokinesis and the presence of several cytokinesis bridges only in half of the colon cancer cell lines tested, although all of them lacked expression of Spartin. The MSS and MSI developmental pathways are characterized by accumulation of distinct aberrations. The latter group, which accounts for approximately $15 \%$ of sporadic colorectal cancers, has a defect in the DNA mismatch repair system leading to frequent aberrations in microsatellites, but the tumors are typically near diploid. On the other hand, the majority of sporadic colorectal carcinomas $(\sim 85 \%)$ are microsatellite stable (MSS), but typically contain multiple chromosomal aberrations (that is, aneuploid). The molecular basis of the MSS phenotype in Spartindeficient colon cancer cells is not clarified, but a possible mechanism is that aberrant cytokinesis may lead to chromosome-containing intercellular bridges that eventually lead to reduced fidelity in chromosome distribution (Steigemann et al., 2009).

A number of additional roles have been described for the Spartin protein, including in endocytosis and degradation of epidermal growth factor receptor (Bakowska et al., 2007), and regulation of the size and number of lipid droplets (Eastman et al., 2009; Hooper et al., 2010). Cells lacking expression of Spartin will therefore suffer aberrations in several cellular mechan- 
isms, possibly creating a cellular 'lability', which makes them more prone to accumulate additional aberrations. In the present study, we showed that hypermethylation of $S P G 20$ and subsequent loss of gene and protein expression is an early and frequent event in colorectal tumorigenesis. SPG20 hypermethylation was present in the majority of early precursor lesions, adenomas. Only a small fraction of these benign tumors, (the ones that accumulate aberrations necessary for malignant transformation) will progress into malignant carcinomas.

In summary, detection and quantification of SPG20 promoter methylation is a highly specific and sensitive biomarker for colorectal tumors. Analyses of a pilot of stool samples from colon cancer patients indicate that $S P G 20$ promoter methylation can be detected in such samples, which is very promising for further development of $S P G 20$ as a biomarker in non-invasive tests. This should be explored further using stool or plasma samples from large test groups. We further show that promoter hypermethylation of SPG20 is associated with loss of gene expression and subsequent lack of Spartin protein. The presence of aberrant cytokinesis profiles as well as convoluted midbodies in Spartin-depleted HeLa and hTERT RPE-1 cells, and Spartin-deficient 'slower' growing colon cancer cell lines reveals a function for the Spartin protein also in cytokinesis. Our data not only identify a promising biomarker for colorectal cancer, but also reveal a cellular mechanism that could be involved in the early carcinogenic process.

\section{Materials and methods}

Cancer cell lines, patients and tissue samples

The present study includes 20 colon cancer cell lines, CRL-1790 and hTERT RPE-1 (epithelial lines from normal colon and retina, respectively), and 30 cell lines representative of breast, cervix, gastric, kidney, ovary, pancreas, prostate and uterus cancer (see Supplementary information for details).

Six of the colon cancer cell lines (MSI: HCT15, RKO and SW48; MSS: HT29, LS1034 and SW480) were subjected to epigenetic treatment using the demethylating drug AZA $(1 \mu \mathrm{M}$ for $72 \mathrm{~h}$; Sigma-Aldrich, St Louis, MO, USA), the histone deacetylase inhibitor TSA $(0.5 \mu \mathrm{M}$ for $12 \mathrm{~h}$; Sigma-Aldrich $)$ and a combination of both drugs $(1 \mu \mathrm{M}$ AZA for $72 \mathrm{~h}$ and $0.5 \mu \mathrm{M}$ TSA added the last $12 \mathrm{~h}$ ). In parallel, the same cell lines were cultured without treatment for $72 \mathrm{~h}$.

Four hundred tissue samples divided between test and validation sets were analyzed, including 179 colorectal carcinomas (47 MSI; 26\% and 132 MSS; 74\%), 111 adenomas and 110 normal mucosa samples. The test and validation sets were from clinically independent sample series (Supplementary Table S1). Additionally, normal mucosa samples $(n=105)$ taken in distance from the carcinomas included in the validation set were analyzed.

Paired colorectal carcinoma and stool samples from nine patients were analyzed, including a stool sample taken proximally from the colorectal carcinoma (during surgery; see Supplementary information).

\section{Qualitative and quantitative MSP}

Bisulfite-treated DNA from cancer cell lines was analyzed by qualitative methylation-specific PCR (MSP), whereas clinical samples were analyzed by qMSP (see Supplementary Table S2 for the primer sequences and Supplementary information for assay details).

\section{Bisulfite sequencing}

Bisulfite sequencing primers were designed using Methyl Primer Express v1.0 (Applied Biosystems, Carlsbad, CA, USA) and purchased from MedProbe. Two fragments were designed in order to fully cover the area amplified by MSP and qMSP (Supplementary Figure S1). See Supplementary Table S2 for primer sequences. A representative promoter region of $S P G 20$ was subjected to direct bisulfite sequencing in 20 colon cancer cell lines.

\section{Real-time quantitative gene expression analysis}

Forty-nine cell lines, 111 colorectal carcinomas (100 methylated and 11 unmethylated) and 41 normal mucosa samples (all unmethylated) were subjected to quantitative mRNA analysis. Prior to the analysis, the high-capacity cDNA archive kit (Applied Biosystems) was used to convert total RNA to cDNA. cDNA was amplified in separate 384-wells plates using the Hs00374834_m1 assay for SPG20, and Hs99999903_m1 and Hs99999908_m1 for the endogenous controls, $A C T B$ and GUSB, respectively. Samples were analyzed in triplicates according to the manufacturer's protocol using the 7900HT Sequence Detection System (Applied Biosystems). A standard curve was generated from a serial dilution of cDNA generated from the human universal reference RNA (Agilent Technologies, Santa Clara, CA, USA). To adjust for potential variation in the template amount in the individual reactions, the median gene expression value from the $S P G 20$ assay was normalized against the mean value of the endogenous controls.

\section{Antibodies and fluorescent probes}

Rabbit anti-Spartin was purchased from Protein Tech Group Inc. (Chicago, IL, USA). Rabbit anti-Aurora-B was from Abcam (Cambridge, UK). Mouse anti- $\alpha$-tubulin, $\gamma$-tubulin and Hoechst 33342 were from Sigma-Aldrich.

\section{siRNA transfection}

The non-targeting RNA duplex and the Spartin siRNA duplexes were from Invitrogen (Carlsbad, CA, USA) and have been published before (see references Cabezas et al. (2005) and Bakowska et al. (2007), respectively). HeLa and hTERT RPE-1 cells grown in six-well plates were transfected with Lipofectamin RNAiMax (Invitrogen) and $50 \mathrm{~nm}$ siRNA the same day as they were seeded. The next day they were split and seeded out onto coverslips where they could grow for 2,3 or 4 days. Separate transfections were performed for the different time points.

\section{Confocal fluorescence microscopy}

The cells were permeabilized or not with $0.05 \%$ saponin, fixed with $3 \%$ paraformaldehyde (PFA) or methanol, and stained for fluorescence microscopy as described by Simonsen et al. (1998). Coverslips were analyzed with a Zeiss LSM 710 laser scanning system equipped with an invert Axio Observer.Z1 microscope using a Plan-Apochromat X63/1.4 Oil DICII objective. Pictures were analyzed using the ZEN 2009 Light Edition software.

High-content analysis of growth rate and cytokinesis profiles Cells fixed in 3\% PFA were stained for $\alpha$-tubulin, Aurora-B and DNA. Images were taken with an Olympus Scan R microscope and analyzed as previously described by Sagona et al. (2010). For relative cell growth analysis, the number of cells obtained from the CRL-1790 normal colon epithelial cell line was set to 1 for each experiment. 


\section{Ethics}

All samples belong to approved research biobanks registered at the Norwegian Institute of Public Health. The present research project has been approved by the Regional Ethics Committee and National Data Inspectorate of Norway.

\section{Statistical analysis}

SPSS v16.0 was used for statistical analyses. Pearson's $\chi^{2}$ and Fisher's exact tests were used for categorical variables. The relationship between promoter methylation status and gene expression was analyzed using $t$-test (cell lines) and Mann-Whitney (tissue) test. ROC curves were generated using percentage methylated reference values. All $P$-values derived from two-tailed tests.

\section{Conflict of interest}

A patent application has been filed covering SPG20 as a biomarker for early detection of colorectal cancer
(Patent Cooperation Treaty; international number PCT/ EP2008/052156).

\section{Acknowledgements}

We are grateful to Dr Hans Geir Eiken for providing expertise on stool DNA extraction and Hilde Honne, Mette Eknaes, Merete Hektoen and Kim Andresen for technical assistance. The breast cancer and pancreatic cancer cell lines were provided by Dr Anne Kallioniemi (Tampere University Hospital, Finland). The non-commercially available colon cancer cell lines were provided by Dr Richard Hamelin (INSERM, Paris, France). Financial support: This study is supported by grants from the Norwegian Cancer Society: GEL as a senior research fellow (PR-2008-0163), CR as a postdoctoral fellow (PR-2007-0063, HS) and SAD as a PhD student (PR-2006-0442, RAL), long-term grant (A95068, RAL). HS is supported by an Advanced Grant from the European Research Council.

\section{References}

Bakowska JC, Jenkins R, Pendleton J, Blackstone C. (2005). The Troyer syndrome (SPG20) protein spartin interacts with Eps15. Biochem Biophys Res Commun 334: 1042-1048.

Bakowska JC, Jupille H, Fatheddin P, Puertollano R, Blackstone C. (2007). Troyer syndrome protein spartin is mono-ubiquitinated and functions in EGF receptor trafficking. Mol Biol Cell 18: 1683-1692.

Bakowska JC, Wang H, Xin B, Sumner CJ, Blackstone C. (2008). Lack of spartin protein in Troyer syndrome: a loss-of-function disease mechanism? Arch Neurol 65: 520-524.

Bu Y, Yang Z, Li Q, Song F. (2008). Silencing of polo-like kinase (Plk) 1 via siRNA causes inhibition of growth and induction of apoptosis in human esophageal cancer cells. Oncology 74: 198-206.

Cabezas A, Bache KG, Brech A, Stenmark H. (2005). Alix regulates cortical actin and the spatial distribution of endosomes. J Cell Sci 118: $2625-2635$.

Carmena M, Earnshaw WC. (2003). The cellular geography of aurora kinases. Nat Rev Mol Cell Biol 4: 842-854.

Chen WD, Han ZJ, Skoletsky J, Olson J, Sah J, Myeroff L et al. (2005). Detection in fecal DNA of colon cancer-specific methylation of the nonexpressed vimentin gene. J Natl Cancer Inst 97: $1124-1132$

Ciccarelli FD, Proukakis C, Patel H, Cross H, Azam S, Patton MA et al. (2003). The identification of a conserved domain in both spartin and spastin, mutated in hereditary spastic paraplegia. Genomics 81: 437-441.

Connell JW, Lindon C, Luzio JP, Reid E. (2009). Spastin couples microtubule severing to membrane traffic in completion of cytokinesis and secretion. Traffic 10: 42-56.

Devos T, Tetzner R, Model F, Weiss G, Schuster M, Distler J et al. (2009). Circulating methylated SEPT9 DNA in plasma is a biomarker for colorectal cancer. Clin Chem 55: 1337-1346.

Eastman SW, Yassaee M, Bieniasz PD. (2009). A role for ubiquitin ligases and Spartin/SPG20 in lipid droplet turnover. J Cell Biol 184: 881-894.

Edwards TL, Clowes VE, Tsang HT, Connell JW, Sanderson CM, Luzio JP et al. (2009). Endogenous spartin (SPG20) is recruited to endosomes and lipid droplets and interacts with the ubiquitin E3 ligases AIP4 and AIP5. Biochem J 423: 31-39.

Errico A, Claudiani P, D'Addio M, Rugarli EI. (2004). Spastin interacts with the centrosomal protein NA14, and is enriched in the spindle pole, the midbody and the distal axon. Hum Mol Genet 13: 2121-2132.

Feinberg AP, Ohlsson R, Henikoff S. (2006). The epigenetic progenitor origin of human cancer. Nat Rev Genet 7: 21-33.
Ferlay J, Autier P, Boniol M, Heanue M, Colombet M, Boyle P. (2007). Estimates of the cancer incidence and mortality in Europe in 2006. Ann Oncol 18: 581-592.

Fujiwara T, Bandi M, Nitta M, Ivanova EV, Bronson RT, Pellman D. (2005). Cytokinesis failure generating tetraploids promotes tumorigenesis in p53-null cells. Nature 437: 1043-1047.

Ganem NJ, Storchova Z, Pellman D. (2007). Tetraploidy, aneuploidy and cancer. Curr Opin Genet Dev 17: 157-162.

Goh L, Murphy SK, Muhkerjee S, Furey TS. (2007). Genomic sweeping for hypermethylated genes. Bioinformatics 23: 281-288.

Hazan J, Fonknechten N, Mavel D, Paternotte C, Samson D, Artiguenave $\mathrm{F}$ et al. (1999). Spastin, a new AAA protein, is altered in the most frequent form of autosomal dominant spastic paraplegia. Nat Genet 23: 296-303.

Hooper C, Puttamadappa SS, Loring Z, Shekhtman A, Bakowska JC. (2010). Spartin activates atrophin-1-interacting protein 4 (AIP4) E3 ubiquitin ligase and promotes ubiquitination of adipophilin on lipid droplets. BMC Biol 8: 72 .

Jemal A, Siegel R, Ward E, Hao Y, Xu J, Thun MJ. (2009). Cancer statistics, 2009. CA Cancer J Clin 59: 225-249.

Jones PA, Baylin SB. (2002). The fundamental role of epigenetic events in cancer. Nat Rev Genet 3: 415-428.

Kim MS, Lee J, Sidransky D. (2010). DNA methylation markers in colorectal cancer. Cancer Metastasis Rev 29: 181-206.

Kron K, Pethe V, Briollais L, Sadikovic B, Ozcelik H, Sunderji A et al. (2009). Discovery of novel hypermethylated genes in prostate cancer using genomic $\mathrm{CpG}$ island microarrays. PLOS ONE 4: e4830.

Lengauer C, Kinzler KW, Vogelstein B. (1997). Genetic instability in colorectal cancers. Nature 386: 623-627.

Lind GE, Ahlquist T, Lothe RA. (2007). DNA hypermethylation of MAL: a promising diagnostic biomarker for colorectal tumors. Gastroenterology 132: 1631-1632.

Lind GE, Kleivi K, Meling GI, Teixeira MR, Thiis-Evensen E, Rognum TO et al. (2006). ADAMTS1, CRABP1, and NR3C1 identified as epigenetically deregulated genes in colorectal tumorigenesis. Cell Oncol 28: 259-272.

Lu J, Rashid F, Byrne PC. (2006). The hereditary spastic paraplegia protein spartin localises to mitochondria. J Neurochem $\mathbf{9 8}$ : 1908-1919.

Milewska M, McRedmond J, Byrne PC. (2009). Identification of novel spartin-interactors shows spartin is a multifunctional protein. J Neurochem 111: 1022-1030. 
Müller HM, Oberwalder M, Fiegl H, Morandell M, Goebel G, Zitt M et al. (2004). Methylation changes in faecal DNA: a marker for colorectal cancer screening? Lancet 363: 1283-1285.

Murata-Hori M, Tatsuka M, Wang YL. (2002). Probing the dynamics and functions of aurora B kinase in living cells during mitosis and cytokinesis. Mol Biol Cell 13: 1099-1108.

Patel H, Cross H, Proukakis C, Hershberger R, Bork P, Ciccarelli FD et al. (2002). SPG20 is mutated in Troyer syndrome, an hereditary spastic paraplegia. Nat Genet 31: 347-348.

Pointis G, Gilleron J, Carette D, Segretain D. (2010). Physiological and physiopathological aspects of connexins and communicating gap junctions in spermatogenesis. Philos Trans $R$ Soc Lond B Biol Sci 365: 1607-1620.

Proukakis C, Cross H, Patel H, Patton MA, Valentine A, Crosby AH. (2004). Troyer syndrome revisited. A clinical and radiological study of a complicated hereditary spastic paraplegia. J Neurol 251: 1105-1110.

Renvoise B, Parker RL, Yang D, Bakowska JC, Hurley JH, Blackstone C. (2010). SPG20 protein spartin is recruited to midbodies by ESCRT-III protein Ist1 and participates in cytokinesis. Mol Biol Cell 21: 3293-3303.

Robay D, Patel H, Simpson MA, Brown NA, Crosby AH. (2006). Endogenous spartin, mutated in hereditary spastic paraplegia, has a complex subcellular localization suggesting diverse roles in neurons. Exp Cell Res 312: 2764-2777.

Sagona AP, Nezis IP, Pedersen NM, Liestol K, Poulton J, Rusten TE et al. (2010). PtdIns(3)P controls cytokinesis through KIF13Amediated recruitment of FYVE-CENT to the midbody. Nat Cell Biol 12: 362-371.

Sagona AP, Stenmark H. (2010). Cytokinesis and cancer. FEBS Lett 584: $2652-2661$.
Simonsen A, Bremnes B, Ronning E, Aasland R, Stenmark H. (1998). Syntaxin-16, a putative Golgi t-SNARE. Eur J Cell Biol 75: 223-231.

Steigemann P, Gerlich DW. (2009). Cytokinetic abscission: cellular dynamics at the midbody. Trends Cell Biol 19: 606-616.

Steigemann P, Wurzenberger C, Schmitz MH, Held M, Guizetti J, Maar S et al. (2009). Aurora B-mediated abscission checkpoint protects against tetraploidization. Cell 136: 473-484.

Tsang HT, Edwards TL, Wang X, Connell JW, Davies RJ, Durrington HJ et al. (2009). The hereditary spastic paraplegia proteins NIPA1, spastin and spartin are inhibitors of mammalian BMP signalling. Hum Mol Genet 18: 3805-3821.

Ventelä S, Toppari J, Parvinen M. (2003). Intercellular organelle traffic through cytoplasmic bridges in early spermatids of the rat: mechanisms of haploid gene product sharing. Mol Biol Cell 14: 2768-2780.

Vidulescu C, Clejan S, O'connor KC. (2004). Vesicle traffic through intercellular bridges in DU 145 human prostate cancer cells. $J$ Cell Mol Med 8: 388-396.

Zhang W, Bauer M, Croner RS, Pelz JO, Lodygin D, Hermeking H et al. (2007). DNA stool test for colorectal cancer: hypermethylation of the secreted frizzled-related protein-1 gene. Dis Colon Rectum 50: $1618-1626$

Zitt M, Zitt M, Muller HM. (2007). DNA methylation in colorectal cancer-impact on screening and therapy monitoring modalities? Dis Markers 23: 51-71.

This work is licensed under the Creative Commons
Attribution-NonCommercial-No Derivative
Works 3.0 Unported License. To view a copy of this license,
visit http://creativecommons.org/licenses/by-nc-nd/3.0/

Supplementary Information accompanies the paper on the Oncogene website (http://www.nature.com/onc) 